\title{
Spatial image compounding applied to a Phase Coherence corrected UT-PA technique for inspecting nuclear components of coarse-grained structure
}

\author{
Jose Brizuela ${ }^{1}$, Pablo Katchadjian ${ }^{2, \text { a) }}$, Alejandro Garcia ${ }^{2}$, Carlos Desimone ${ }^{2}$ \\ ${ }^{1}$ Consejo Nacional de Investigaciones Científicas y Técnicas, Buenos Aires, Argentina. \\ ${ }^{2}$ Comisión Nacional de Energía Atómica, Dept. ENDE - INEND, Buenos Aires, Argentina. \\ a)katcha@cnea.gov.ar
}

\begin{abstract}
The aim of this work is to obtain a C-Scan view of an austenitic stainless steel weld from a nuclear use pipe. In order to obtain this result Sectorial Scans (S-Scan) from both sides of the weld are obtained by Ultrasonic Phase Array (UT-PA). Then, spatial image compounding is performed to generate a single image from the S-Scans acquired at the same circumferential position of the transducer.

These joints have a coarse grain structure which significantly reduce the transmission of the ultrasonic wave due to attenuation characteristics and backscattered noise from microstructures inside the material. For this reason, phase coherence imaging technique has been also applied to reduce the structural noise and improve the image quality.

To verify detected defects, and given the impossibility of cutting the component, gammagraphy were performed with Co60.
\end{abstract}

\section{INTRODUCTION}

This work describes an ultrasonic inspection procedure designed to verify coarse-grained structure materials which are commonly used in nuclear power plants (NPP) components.

In order to obtain this result, Sectorial Scans (S-Scan) from both sides of the weld are obtained by Ultrasonic Phase Array (UT-PA). Then, spatial image compounding [1] is performed to generate a single image from the SScans which were acquired at the same circumferential position of the transducer. This main signal processing involves a bilinear interpolation [2] that is used for the recombination of echoes from the same reflectors obtained from different propagation paths considering only the amplitude information. This simplifies the interpretation of the resulting images and allows producing a corrected C-Scan view.

Coarse grain structure of these materials significantly reduces the transmission of ultrasonic waves due to attenuation characteristics and backscattered noise from microstructures inside the material. For this reason, phase coherence imaging technique has been studied and used for reducing the structural noise and improve the image quality.

To verify detected defects, and given the impossibility to destructively analyze the weld, gammagraphy were performed with Co60 source. 

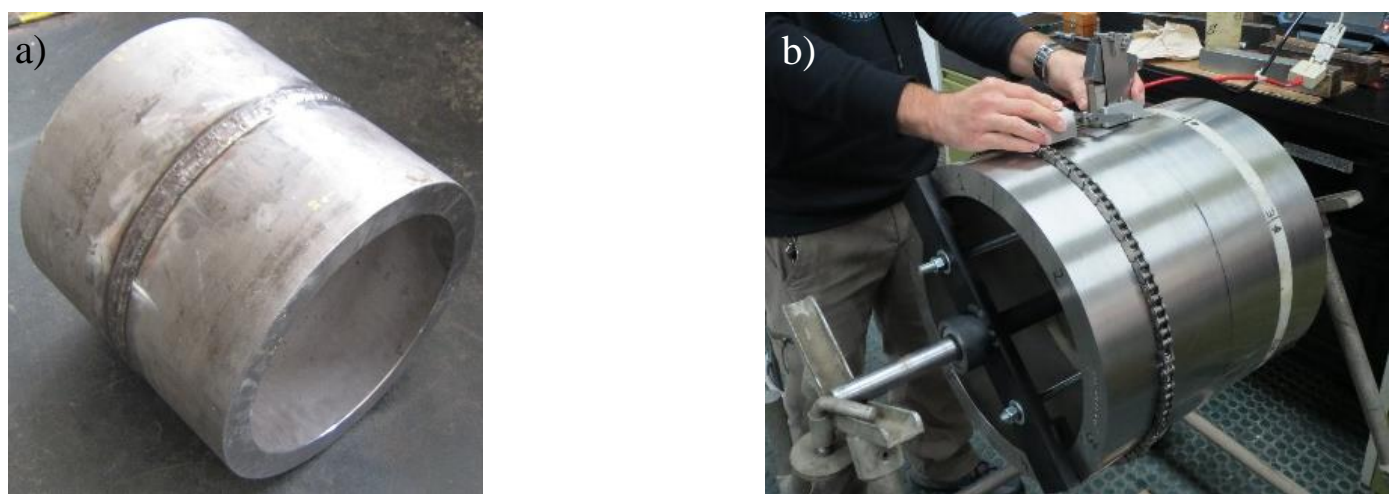

FIGURE 1. a) Stainless steel tube from NPP moderator system and b) Acquisition support structure.

\section{Phase coherence technique}

The phase coherence technique is based on analyzing the phase information contained in each received signal in order to calculate a phase coherence factor (PCF) that measures the phase dispersion of the aperture data. This factor quantifies the focusing quality, ie.: if the echo was generated by a real reflector, located at the focal point, it will be weighted close to 1 , but if the echo comes from anywhere else or if it's just noise, PCF will be close to 0 . This technique significantly improves the resolution and dynamic range of the image [3].

In materials that exhibit grain noise due to internal crystal structure (ie. austenitic stainless steel), this technique succeeds in reducing noise, since signals produced by grains are not fully coherent and they will be cancelled [4].

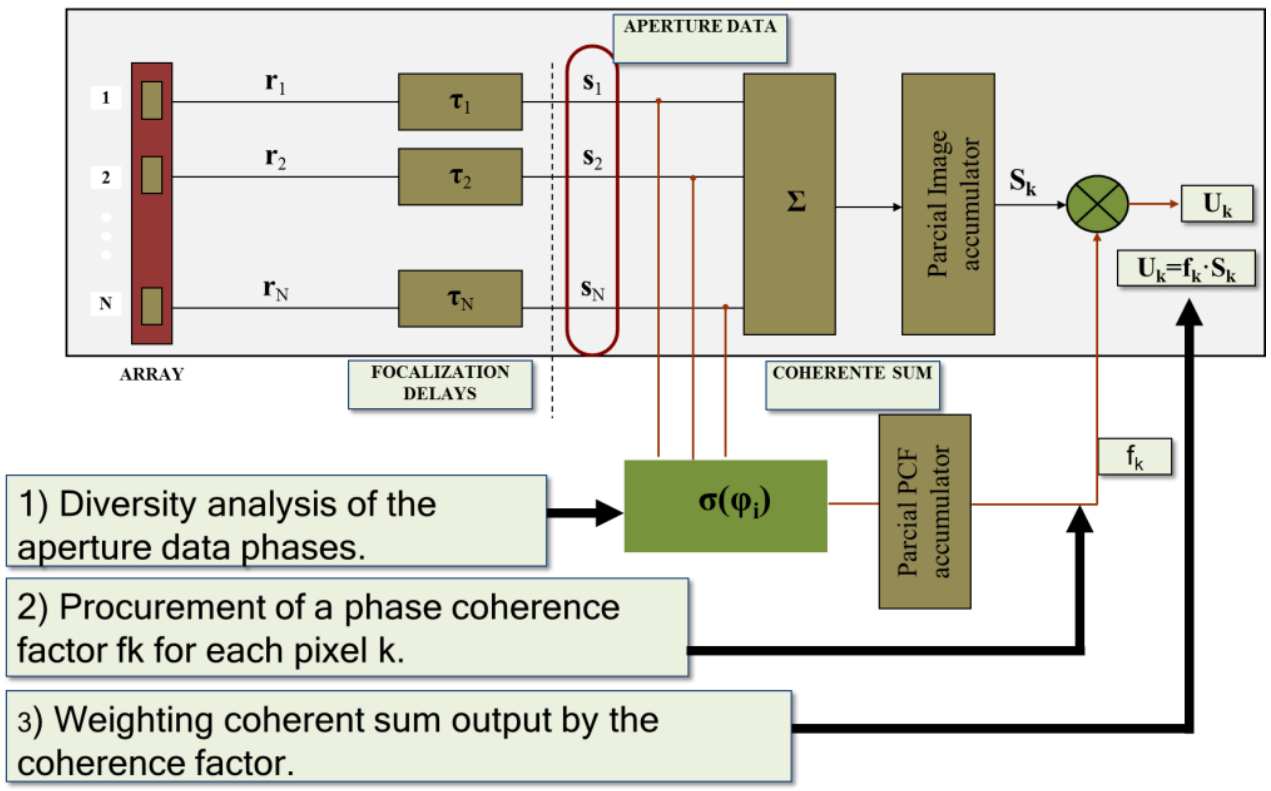

FIGURE 2. Phase Coherence implementation diagram.

\section{EXPERIMENTAL DETAIL}

The test piece used was a Pipe welding from NPP moderator system made of austenitic stainless steel grade 1.4550 , with a thickness $=40 \mathrm{~mm}$ and a $\varnothing$ ext $=428 \mathrm{~mm}$ (fig. 1 -a). The outer diameter weld reinforcement was removed through a mechanical machining to simulate real inspection conditions over the component. The test piece was manipulated using an arrangement specially developed for performing the experiments (fig.1-b). 
The weld edge preparation is shown in fig.3-a. The grain structure of the weld profile was analyzed by macrography as can be seen in fig.3-b.

SITAU PA-311 (DASEL, Spain) system was used in joint with a 5MHz array of 128 elements, $10 \mathrm{~mm}$ length in the elevation plane and $0,5 \mathrm{~mm}$ pitch (Imasonic, France). The wedges used had a centre angle of $55^{\circ}$ for transversal wave (T-Wave) and $60^{\circ}$ for longitudinal wave (L-Wave). Circumferential position was acquired with an Olympus Versamouse encoder with a resolution of $8.4 \mathrm{pulses} / \mathrm{mm}$ (fig.3-c).

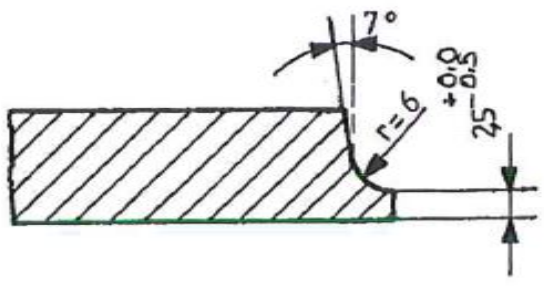

a)

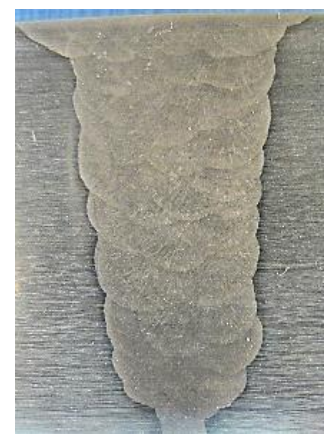

b)

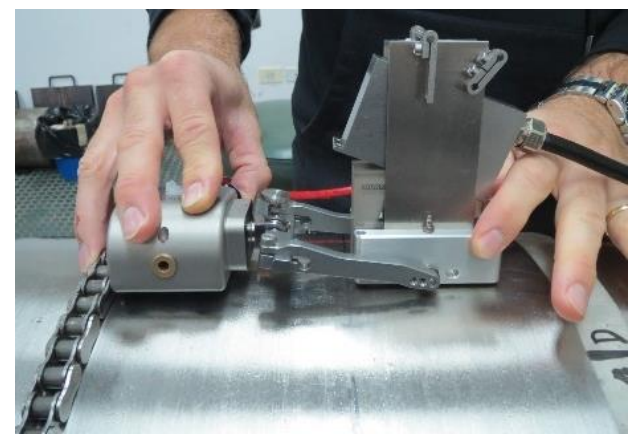

c)

FIGURE 3. a) Weld edge preparation, b) Macrography of the welding and c) Data acquisition system configuration

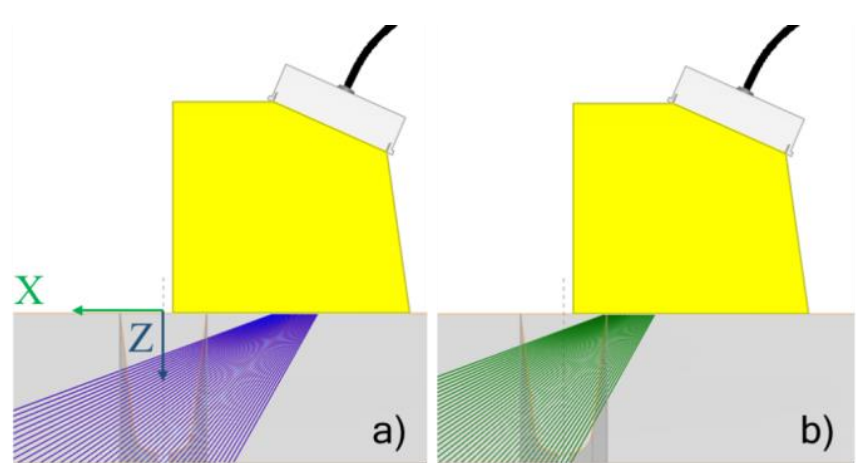

FIGURE 4. a) Scan with elements 1 to 32 and b) elements 33 to 64

\section{Description of the applied technique}

Ultrasonic Phased Array technique was used with both longitudinal and transversal waves to determine the best test conditions. Dynamic depth focusing (DDF) technique was applied to improve the lateral resolution and reduce noise due to beam dispersion [5]. In addition, the phase coherence algorithm was used to improve the SNR of images by computing the PCF factor for each sample of each S-Scan acquired.

The welded joint was inspected from both sides with the transducer in a fixed position and varying the used aperture (elements 1 to 32 , or 33 to 64). The angular range used was $30^{\circ}$ to $70^{\circ}$ with a resolution of $1^{\circ}$ (fig.4). Circumferential scanning resolution was $1 \mathrm{~mm}$ (Y direction) (fig.5-b)

From sectorial scans, a post processing for spatial image compounding was performed [1] to generate side views (D-Scan) and geometrically corrected C scans.

\section{Spatial Image Compounding}

The spatial composition is performed from two S-Scans, one on each side of the weld (fig.5-a). These S-Scans show the ultrasonic data in polar coordinates. Thus, a bilinear interpolation of polar images into a rectangular grid of $n \times m$ pixels is carried on, where $n$ is associated with the axis perpendicular to the weld (X axis) and $m$ to component depth ( $\mathrm{Z}$ axis) (fig.5-b). 
Then, the interpolated images generated for each circumferential position of the weld are merged to produce a compounded image corresponding to each position.

The resulting data cube with $n \times m \times p$ dimension is obtained from all matrices generated in the way described above, where $p$ corresponds to the circumferential position of the weld (Y axis).

Once this data cube is obtained, different geometrically corrected views can be generated (i.e. Side View, CScan, etc.). The region of interest is analyzed by adjusting the depth range of the visualization gate.

a)

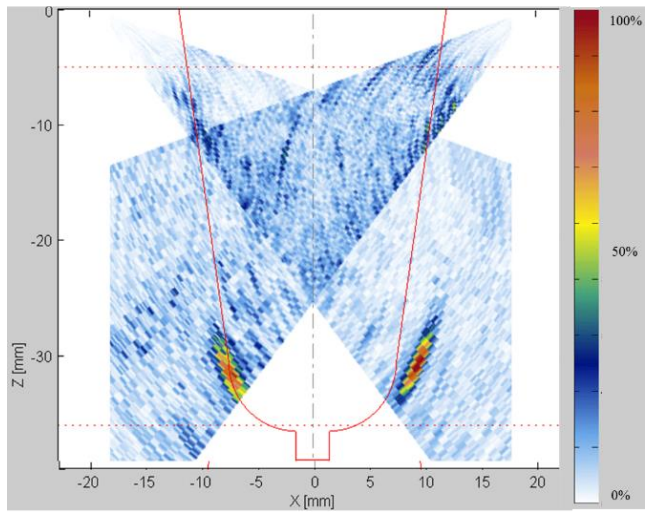

b)

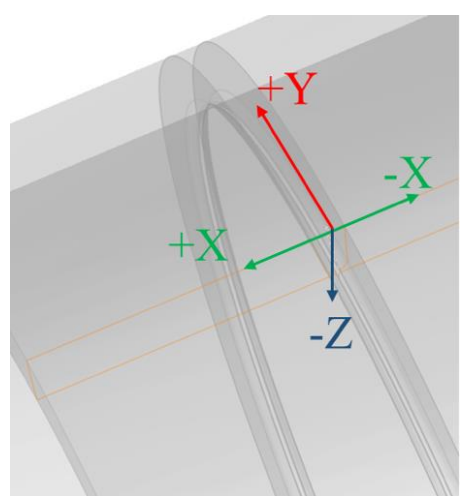

FIGURE 5. a) S-Scans at both sides of the weld and b) Coordinate system used.

\section{Inspection Results}

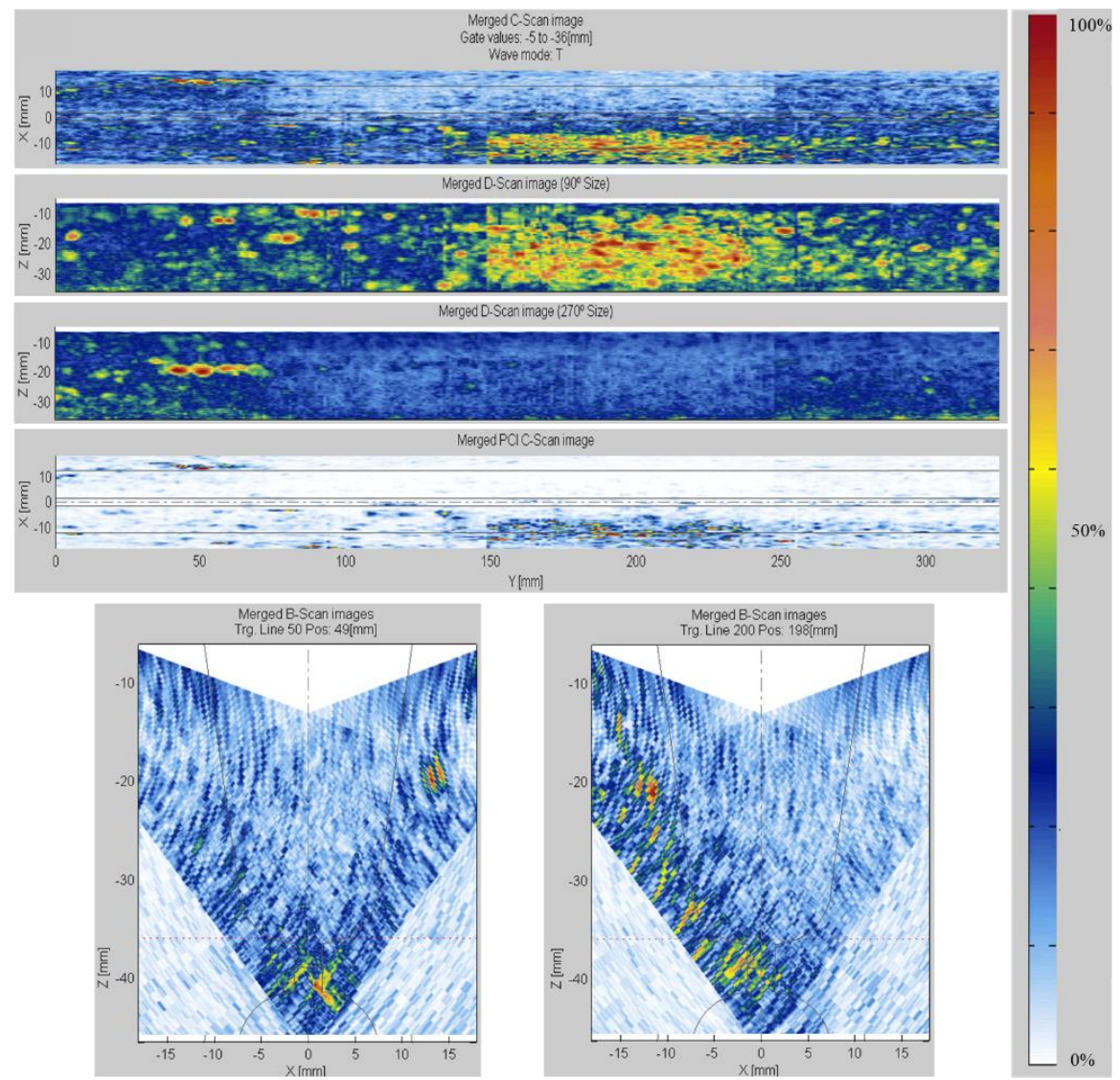

FIGURE 6. Images generated with aperture between elements 1 to 32 (T-Wave) 
Images generated by the described process, with aperture between elements 1 to 32 (fig. 6) and 33 to 64 (fig. 7) are presented. They are shown in this order: C-Scan (top view), D-Scan (side view) between the center of the weld and $-18 \mathrm{~mm}\left(270^{\circ}\right.$ orientation array), D-Scan between the center of the weld and $+18 \mathrm{~mm}\left(90^{\circ}\right.$ orientation array); and C-Scan using phase coherence imaging (PCI). Also, two merged B-Scans are shown in each case at specific positions for better interpretation over the welding mask.

In these image series, several lateral lacks of fusion can be seen, both in the $7^{\circ}$ angled side and curved region of the weld edge.

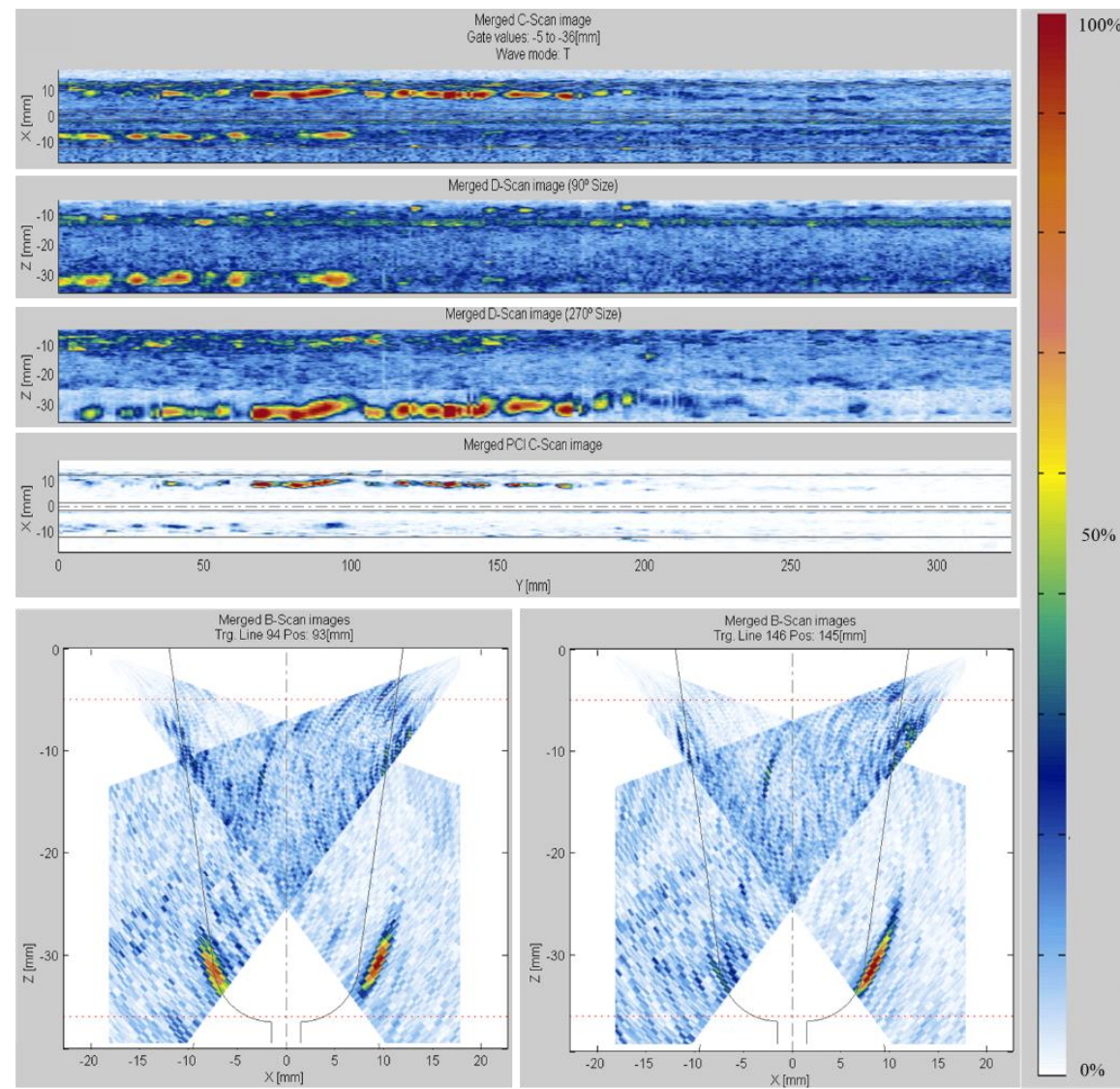

FIGURE 7. Images generated with aperture between elements 33 to 64 (T-Wave)

a)
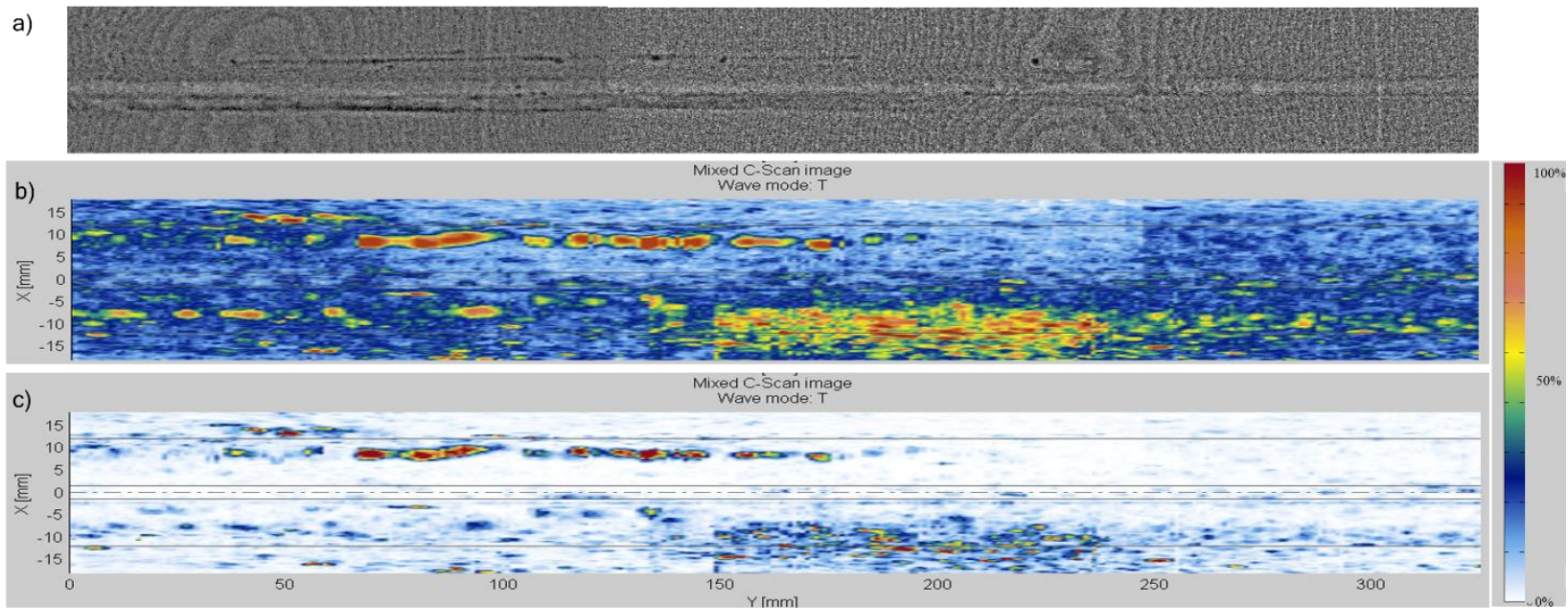

FIGURE 8. a) Co60 gammagraphy, b) merged C-scan without PCI and c) merged C-scan with PCI 
As final result, a merge of the C-scan images taken with apertures between elements 1 to 32 and 33 to 64 was done. Thus, two corrected C-Scan were obtained: with PCI (fig. 8-c) and without PCI (fig. 8-b). Also, the radiographic image (Co60 gammagraphy) of the region of interest (fig. 8-a), digitized, processed and filtered is presented to verify the results obtained by UT-PA.

\section{CONCLUSIONS}

- After the spatial compounding of images, the interpretation of the information obtained is improve, thus facilitating the analysis of data from the weld.

- In the images generated with longitudinal wave, for the same angular range and calibration sensitivity, most of the defects detected with transverse wave cannot be seen.

- It was able to verify the discontinuities observed with Co60 gammagraphy, noting that some defects are best detected by the UT-PA technique proposed.

\section{FUTURE WORKS}

- Apply this technique for inspecting dissimilar metal welds used in nuclear components, i.e.. RPV to Plenum (Nozzle) welding in Argentinian CAREM NPP prototype.

- Destructively study the defects detected to understand the reason they are not equally detected by longitudinal waves under the same conditions.

\section{ACKNOWLEDGMENTS}

The authors thank J. Scopelliti and R. Castillo Guerra for their assistance and dedication

\section{REFERENCES}

1. S. K. Jespersen, J. E. Wilhjelm, H. Sillesen, "Ultrasound Spatial Compound Scanner for Improved Visualization in Vascular Imaging". IEEE Ultrasonic Symposium, vol. 2, pp. 1623-1626 (1998).

2. C. Basoglu, Y. Kim, V. Chalana, "A Real-Time Scan Conversion Algorithm on Commercially Available Microprocessors". Ultrasonic Imaging, vol. 18, pp.: 241-260 (1996).

3. J. Camacho, J. Brizuela, and C. Fritsch, "Grain-noise reduction by phase coherence imaging", Rev. of Quantitative Non Destructive Evaluation, vol. 29, pp. 855-862 (2010).

4. J. Camacho, C. Fritsch, J. Brizuela, and R. González, "Reducción de ruido estructural con Phased Array", VII Congreso Regional de ENDE (CORENDE), Rosario, Argentine (2009).

5. C. Fritsch, M. Parrilla, et al., "The progressive focusing correction technique for ultrasound beamforming". IEEE Trans. UFFC, vol. 53 (10), pp. 1820-1831 (2006). 